\title{
Bladder Calculus Causing Large Bowel Obstruction
}

\author{
Alessandro Bellomo, Morapedi Kwati, Jorge Monzon, Babatunde Ogunrombi
}

Klerksdorp Tshepong Hospital Complex, University of Witwatersrand, South Africa

Correspondence to: Dr. Babatunde Ogunrombi, Benji Oliphant Street, Klerksdorp, North West 2570, South Africa;

email: mogunrom@yahoo.com

\begin{abstract}
Summary
Urinary tract calculi are fairly common, though bladder calculi are less common, accounting for only $5 \%$ of urinary tract calculi. Bladder calculi, particularly large bladder calculi, are usually associated with pathology in the urinary tract. Such pathologies include urethral strictures, bladder neck contractures, benign prostatic hypertrophy, neurogenic bladder and foreign bodies in the bladder. Bladder calculi are more common in men than in women. Bladder calculi causing bowel obstruction have been documented in the literature before but they remain very rare.
\end{abstract}

\section{Introduction}

Bladder calculi, particularly large bladder calculi, are usually associated with pathology in the urinary tract (1). Such pathology includes urethral strictures, bladder neck contractures, benign prostatic hypertrophy (BPH), neurogenic bladder and foreign bodies in the bladder. However, bladder calculi are a very unusual cause of intestinal obstruction and are rarely reported in the literature (2). To our knowledge, this is the first case of a large bladder calculus from a migrated Double-J stent causing large bowel obstruction.

\section{Case report}

A 20-year-old male presented with a 5-day history of generalized abdominal pain and obstipation, as well as 1-day history of vomiting. The patient was known to have had a previous exploratory laparotomy for a penetrating abdominal injury 5 years previously, during which he sustained a splenic laceration, perforations of the transverse colon and left hemi diaphragm and a left-side complete ureteric transection. At the initial laparotomy, a splenectomy was done and the perforations were repaired. A primary ureteric anastomosis was performed and a 'Double-J' stent was left in situ. The patient was subsequently lost to urology follow-up and presented 5 years later with the above symptoms. An impression of an acute abdomen secondary to strangulating adhesive bowel obstruction was made based on the presentation and clinical findings of a distended abdomen, generalized peritonitis and scanty bowel sounds.

Chest X-ray was normal while abdominal X-rays showed distension of multiple loops of small bowel accompanied by distended large bowel and multiple air fluid levels. In addition, the impression of a coiled Double-J stent could be seen in the
Key words: Bladder calculus, Large bowel obstruction.

Ann Afr Surg. 2019; 16(2):82-83

DOI: http://dx.doi.org/10.4314/aas.v16i2.9

Conflicts of Interest: None

Funding: None

(C) 2019 Author. This work is licensed under the Creative Commons Attribution 4.0 International License.

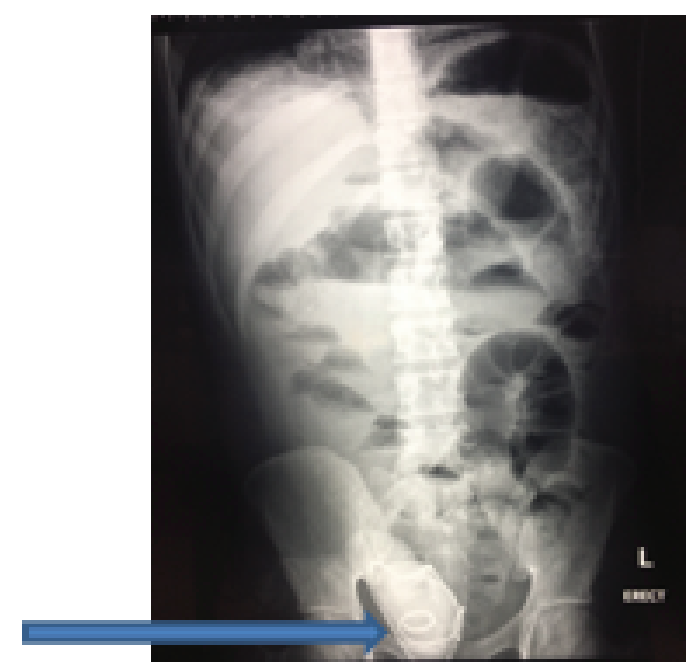

Figure 1. Preoperative erect plain abdominal radiograph showing DJ stent coiled in bladder.

bladder, with a partially radio-opaque mass surrounding it (arrow in Figure 1).

At this stage, a bladder calculus, in favor of adhesions, was not thought to be the cause of the bowel obstruction. Preoperatively, the patient had significantly raised septic markers: white cell count 25,800 cells $/ \mu \mathrm{L}$, range $3,500-10,500 / \mu \mathrm{L}$; C-reactive protein $330 \mathrm{mg} / \mathrm{L}$, range $0-10 \mathrm{mg} / \mathrm{L}$; renal dysfunction - urea $33.4 \mathrm{mmol} / \mathrm{L}$, creatinine $-545 \mathrm{mmol} / \mathrm{L}$ - with a high anion gap metabolic acidosis (AG, 34). Preoperative blood gas confirmed acidosis with a pH of 7.21 and a low hemoglobin of $6.9 \mathrm{~g} / \mathrm{dL}$. The patient was resuscitated with adequate fluid, transfused with a unit of packed red blood cells, and a decision was made for exploratory laparotomy because of the peritonitis. Intraoperatively, multiple small bowel adhesions were found in the left upper quadrant and adhesiolysis was performed; 
however, the distal large bowel continued to be distended. Further exploration showed a large mass was present in the bladder, causing extrinsic compression on the sigmoid colon and exacerbating the obstruction. An extraperitoneal cystotomy was performed and a large calculus $(15 \mathrm{~cm} \times 2.5 \mathrm{~cm}$, estimated weight $200 \mathrm{~g}$ ) was removed (Fig. 2), having calcified around the DJ stent still in situ.

The bladder was repaired in two layers. The initially dusky bowel had regained normal tone and color. No peritoneal soiling was seen. Postoperatively, the patient required 2 days of ventilation to treat the unresolved acidosis. His renal function greatly improved after a few days. A renal ultrasound performed before discharge found bilateral hydronephrosis and hydroureters. His renal function had almost normalized (urea 9 $\mathrm{mmol} / \mathrm{L}$, creatinine $141 \mathrm{mmol} / \mathrm{L}$ ) by the time he was discharged from hospital, in good condition and with a urethral urinary catheter in situ. Biochemical analysis revealed that the stone removed from the patient was composed of carbonate apatite (calcium phosphate).

\section{Discussion}

The presence of large bowel distension, despite adequate adhesiolysis, prompted the search for a distal source of obstruction: a bladder mass was found which had not been suspected preoperatively, causing the extrinsic compression. A case report published in 2004 by Mariappan and Loony was the first documented report of a patient presenting with acute bowel obstruction arising from a large bladder calculus. The authors describe a patient presenting with acute large bowel obstruction secondary to a large bladder calculus, causing extrinsic compression on the rectum, which could be felt on rectal examination. The stone was removed surgically, relieving the symptoms of bowel obstruction (3).

Renal dysfunction in our patient's blood results together with the renal ultrasound findings suggest that the stone was also causing an obstructive uropathy that affected the patient's renal function, which completely resolved once the stone was removed. Minter and Chiovaro documented a patient who had been performing intermittent self-catheterization for benign prostatic hyperplasia. The patient had a large stone that had precipitated around a plastic foreign body (presumably from the self-catheterization) (1).

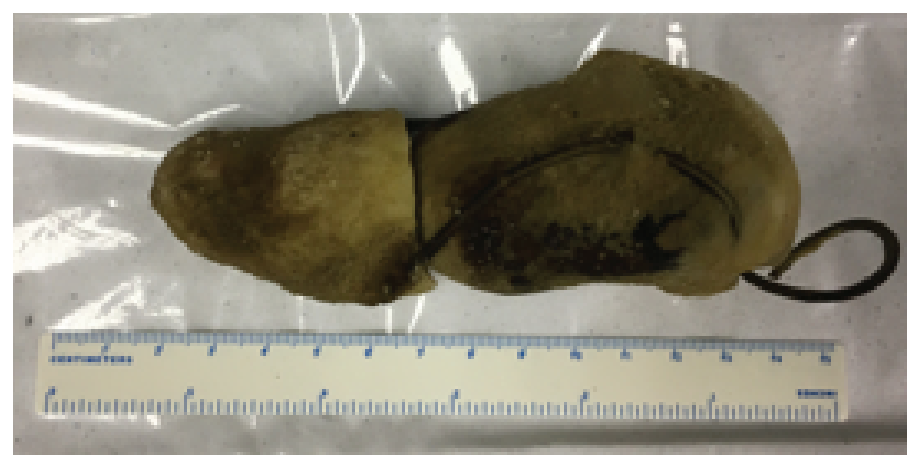

Figure 2. Bladder calculus removed at laparotomy

Foreign bodies in the bladder act as a nidus for crystal precipitation in the bladder once urine reaches a supersaturated state. Stones may be formed from different minerals. The most radio-opaque stones are calcium phosphate and calcium oxalate stones, which also constitute $90 \%$ of urinary tract calculi (4). This is in keeping with the stone retrieved from our patient. Struvite (magnesium-ammonia-phosphate) and cysteine stones are also radio-opaque though less so than calcium-based stones (4). Radiolucent stones comprise matrix stones and those formed from uric acid (4).

\section{Conclusion}

While it remains uncommon, large bladder calculus remains a possible cause of bowel obstruction. Proper review of previous trauma records where available and radiological investigations should be performed on patients with suspected adhesive intestinal obstruction. Renal dysfunction in a young patient with a previous laparotomy should heighten suspicion of a urological cause.

\section{References}

1. Minter J, Chiovaro J. Renal failure with a large bladder calculus related to a foreign body: A case report. Clin Case Rep. 2014; 2:48-50.

2. Hughes KE, Arthur J. An atypical case of large bowel obstruction. J Surg Case Rep. 2013; 2013(6):042.

3. Mariappan P, Loony CW. Vesicolithotomy for acute intestinal obstruction. J Urol. 2004; 171:1233.

4. Hesse A, Heimbach D. Causes of phosphate stone formation and the importance of metaphylaxis by urinary acidification: A review. World J Urol. 1999; 17:308-15. 